\title{
Geometric Eccentricity and the Complexity of Manipulation Plans
}

\author{
A. Frank van der Stappen* Ken Goldberg ${ }^{\dagger} \quad$ Mark H. Overmars*
}

\begin{abstract}
Complexity bounds for algorithms for robotic motion and manipulation can be misleading when they are constructed with pathological 'worst-case' scenarios that rarely appear in practice. Complexity can in some cases be reduced by characterizing nonpathological objects in terms of intuitive geometric properties. In this paper we consider the number of push and push-squeeze actions needed to orient a part without sensors and improve on the upper bound of $O(n)$ for polygonal parts given by Chen and Ierardi in [9]. We define the geometric eccentricity of a planar part based on the aspect ratio of a distinguished bounding box. We show that only $O(1)$ actions are required for parts with non-zero eccentricity. The analysis also applies to curved parts, providing the first complexity bound for non-polygonal parts. Our results also yield new bounds on part feeders that use fences and conveyor belts.
\end{abstract}

\section{Introduction}

Many automated manufacturing processes require parts to be oriented prior to assembly. A part feeder takes in a stream of identical parts in arbitrary orientations and outputs them in a uniform orientation. Part feeders often use data obtained from some kind of sensing to accomplish their task. We consider the problem of sensorless orientation of parts, in which the initial pose of the part is assumed to be unknown. In sensorless manipulation, parts are positioned and/or oriented using passive mechanical compliance. The input is a description of part shape and the output is a sequence of open-loop actions that moves a part from an unknown initial pose into a unique final pose $[2,6,7,8,9,10,11,16,17,20]$.

Goldberg [11] considered the problem of orienting (feeding) polygonal parts using a paralleljaw gripper. A parallel-jaw gripper consists of a pair of flat parallel jaws that can close in the direction orthogonal to the jaws, allowing it to squeeze the part. Figure 1 shows a (frictionless) parallel-jaw gripper. Let $N$ be the length of the shortest sequence of gripper actions that will orient the part up to symmetry. Goldberg showed that $N$ is $O\left(n^{2}\right)$ for polygonal parts with $n$ vertices, and gave an algorithm for finding the shortest squeeze plan. Based on a large number of pseudo-random trials, he conjectured that $N$ is $O(n)$.

Chen and Ierardi [9] proved Goldberg's conjecture using a different planning algorithm. They showed that plans generated by their algorithm, which does not necessarily find the shortest plan, require at most $2 n-1$ actions, thereby proving the $O(n)$ bound on the length of the shortest plan. Chen and Ierardi also construct pathological polygons where $N$ is $\Omega(n)$.

\footnotetext{
*Department of Computer Science, Utrecht University, PO Box 80089, 3508 TB Utrecht, The Netherlands.

${ }^{\dagger}$ Industrial Engineering and Operations Research, University of California at Berkeley, Berkeley, CA 94720, USA.
} 

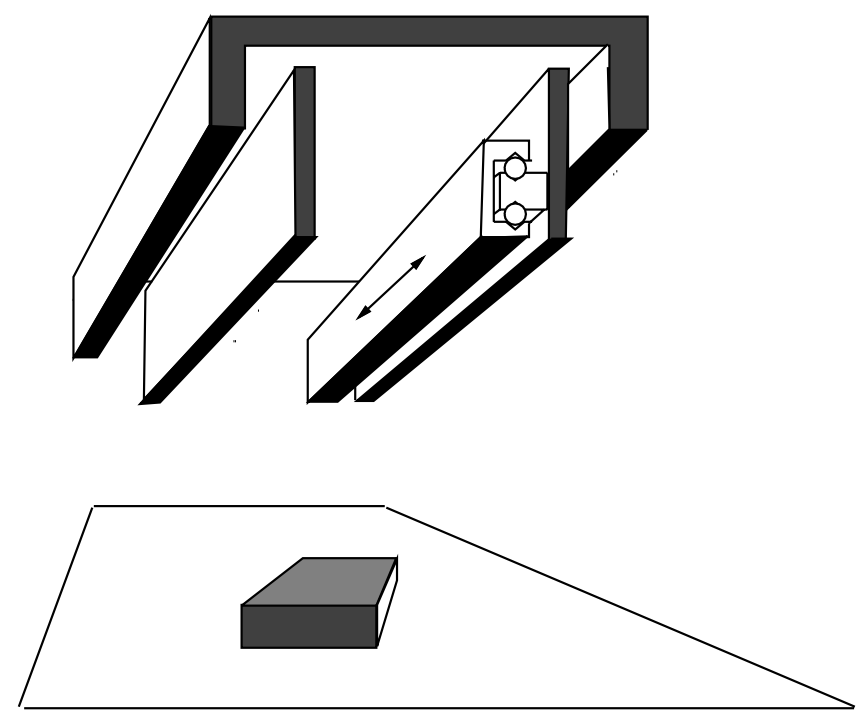

Figure 1: A (frictionless) parallel-jaw gripper positioned over a part.

Such pathological polygons are 'fat' (approximately circular), while $N$ is almost always small for 'thin' parts. Consider the two parts shown in Figure 2. Imagine grasping part A. Regardless of the orientation of the gripper, we expect the part to be squeezed into an orientation in which its longest edge is flushed against a jaw of the gripper. Hence, the number of possible orientations of the part (relative to the gripper) after a single application of the gripper is very small. Part $B$ can end up with any of its $n$ edges against a gripper jaw; the number of possible orientations after a single application of the gripper is considerably higher than in the case of the thin part. In general, we observe that thin parts are easier to orient than fat ones.

The theoretical analysis in this paper confirms this intuition. To formalize our intuition about fatness, we define the geometric eccentricity of a planar part based on the aspect ratio of a distinguished bounding box. We show that only $O(1)$ actions are required for parts with non-zero eccentricity. The analysis also applies to curved parts and provides the first complexity bound for non-polygonal parts [17].

These results for part orienting are in contrast to computational geometric results in robot motion planning, hidden surface removal, depth orders, motion planning, point location, and range searching, where fatness often reduces combinatorial [3, 14, 18] and algorithmic $[1,4,12,15,19]$ complexities. For part orienting, we show that thinness reduces complexity.

Our results have implications for several other sensorless part feeders that use push-like actions to orient a part. One such part feeder aligns parts as they move down a conveyor belt and slide along a sequence of fences placed along the belt $[8,16]$. Because fence design can be regarded as finding a constrained sequence of push actions [5, 20], we find that a constant number of fences suffices to orient parts with non-zero eccentricity.

Akella et al. [2] explore a part feeder (1JOC) consisting of a conveyor belt and a single rotational fence. The fence repeatedly catches the part after which it uses its rotational degree 

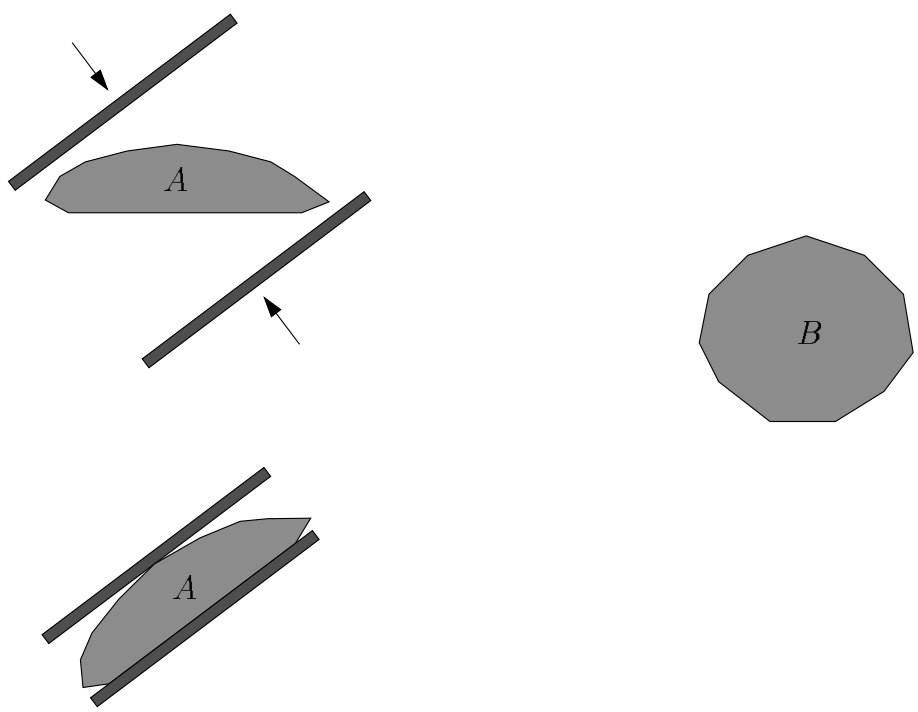

Figure 2: Both polygonal parts have $n=11$, but part $A$ is thin, while $B$ is fat. For part $A$, $N=1$, whereas $N>10$ for part $B$ since it has many edges that can be stable after the first action.

of freedom to reorient the part while transporting it back along the belt (allowing it to be caught again by the fence). As finding a sequence of fence rotations is equivalent to finding a push plan, we conclude that a constant number of catches and reorientations by the fence suffices to orient parts with non-zero eccentricity.

We first prove the result for the case where the actions are pushes by a single jaw, and then extend it to push-squeeze actions by a parallel-jaw gripper. In a push-squeeze action, the part is first pushed by a single jaw until it settles, before the second jaw achieves contact and both jaws start to squeeze the part. We prefer this more realistic model of a gripper action over the model of pure squeezing in which both jaws are assumed to achieve contact with the part simultaneously.

This paper is organized as follows. Section 2 is devoted to preliminaries; it discusses the key notions of equilibrium orientation and geometric eccentricity. In Sections 3 and 4, we establish relations between the longest angular interval without equilibrium (push) orientations on the one hand and the number of actions needed to orient a part and the geometric eccentricity of a part on the other hand. In Section 5, we extend the implied relation between eccentricity and the length of push plans to push-squeeze plans and fence design. Section 6 concludes the paper.

\section{Pushing, squeezing, and geometric eccentricity}

Throughout the paper, we assume zero friction between the part and the jaw(s) of the gripper. As a jaw always touches the part at its convex hull, we assume without loss of generality that the part under consideration is convex. Let $P$ be a convex part.

We assume that a fixed coordinate frame is attached to $P$. Directions are expressed relative to this frame. The contact direction of a supporting line $l$ of a part $P$ is uniquely 
defined as the direction of the vector perpendicular to the direction of $l$ and pointing into $P$. The radius function $r:[0,2 \pi) \rightarrow \mathbb{R}^{+}$of a part $P$ with a center-of-mass $c$ maps a direction $\phi$ onto the distance from the center-of-mass $c$ to the supporting line of $P$ with contact direction $\phi$. Recall that the direction $\phi$ is measured with respect to the frame attached to $P$. The diameter function $d:[0,2 \pi) \rightarrow \mathbb{R}^{+}$of a part $P$ maps a direction $\phi$ onto the distance between the supporting line with contact direction $\phi$ and the supporting line parallel to it, which has contact direction $(\phi+\pi) \bmod 2 \pi$. Note that the definition implies that $d(\phi)=d((\phi+\pi) \bmod 2 \pi)$ for all $\phi \in[0,2 \pi)$, hence $d$ is periodic in $\pi$. The radius and the diameter functions are both continuous. The final orientation of a part that is being pushed or squeezed can be determined from the radius and diameter functions.

Throughout this paper, parts are either pushed by a single jaw in a direction perpendicular to that jaw or squeezed by two parallel jaws that move towards each other in a direction perpendicular to both jaws. In most cases, parts will start to rotate when pushed or squeezed. If pushing or squeezing in a certain direction does not cause the part to rotate, then we refer to the corresponding direction as an equilibrium (push or squeeze) direction or orientation. These equilibrium orientations play a key role throughout this paper. If pushing or squeezing does change the orientation, then this rotation changes the orientation of the pushing or squeezing gripper relative to the part. We assume that pushing or squeezing continues until the part stops rotating and settles in a (stable) equilibrium pose.

The push function $p:[0,2 \pi) \rightarrow[0,2 \pi)$ links every orientation $\phi$ to the orientation $p(\phi)$ in which the part $P$ settles after being pushed by a jaw with initial contact direction $\phi$ (relative to the frame attached to $P$ ). The rotation of the part due to pushing causes the contact direction of the jaw to change. The final orientation $p(\phi)$ of the part is the contact direction of the jaw after the part has settled. The equilibrium push directions are the fixed points of the push function $p$. The squeeze function $s:[0,2 \pi) \rightarrow[0,2 \pi)$ links every orientation $\phi$ to the orientation $s(\phi)$ in which the part $P$ settles after being simultaneously pushed by jaws with initial contact directions $\phi$ and $(\phi+\pi) \bmod 2 \pi$. Both jaws are assumed to achieve contact with the part simultaneously. The final orientation $s(\phi)$ of the part equals the contact direction of the former jaw after the part has settled. The equilibrium squeeze directions are the fixed points of the squeeze function $s$. We will occasionally use the term transfer functions as a common denominator for both push and squeeze functions.

The push function $p$ and the squeeze function $s$ consist of steps, which are intervals $I \subset[0,2 \pi)$ for which $p(\phi)=C$ or $s(\phi)=C$ for all $\phi \in I$ and some constant $C \in I$, and ramps, which are intervals $I \subset[0,2 \pi)$ for which $p(\phi)=\phi$ or $s(\phi)=\phi$ for all $\phi \in I$. Note that the ramps are intervals of equilibrium orientations. The steps and ramps of the push function are easily constructed from the radius function $r$, using the points of horizontal tangency, i.e., the points $\phi$ at which $r^{\prime}(\phi)=0$. All points of horizontal tangency in the radius function are fixed points of the push function. In other words, the part will not rotate when pushed in a direction corresponding to a horizontal tangency of the radius function. The orientations of horizontal tangency are the equilibrium push orientations. Notice that angular intervals of constant radius turn up as ramps of the push function. If the part is pushed in a direction corresponding to a point of non-horizontal tangency of the radius function then the part will rotate in the direction in which the radius decreases. The part finally settles in an orientation corresponding to a local minimum of the radius function. As a result, all points in the open interval $I$ bounded two consecutive local maxima of the radius function $r$ map onto the orientation $\phi \in I$ corresponding to the unique local minimum of $r$ on $I$. (Note that $\phi$ itself maps onto $\phi$ because it is a point of horizontal tangency.) This results in the steps of the 
push function. Note that each half-step, i.e., a part of a step on a single side of the $p(\phi)=\phi$, is a (maximal) angular interval without equilibrium push orientation. Besides the steps and ramps there are isolated points satisfying $p(\phi)=\phi$ in the push function, corresponding to local maxima of the radius function. Figure 3 shows an example of a radius function and the corresponding push function. The construction of the steps and ramps of the squeeze function
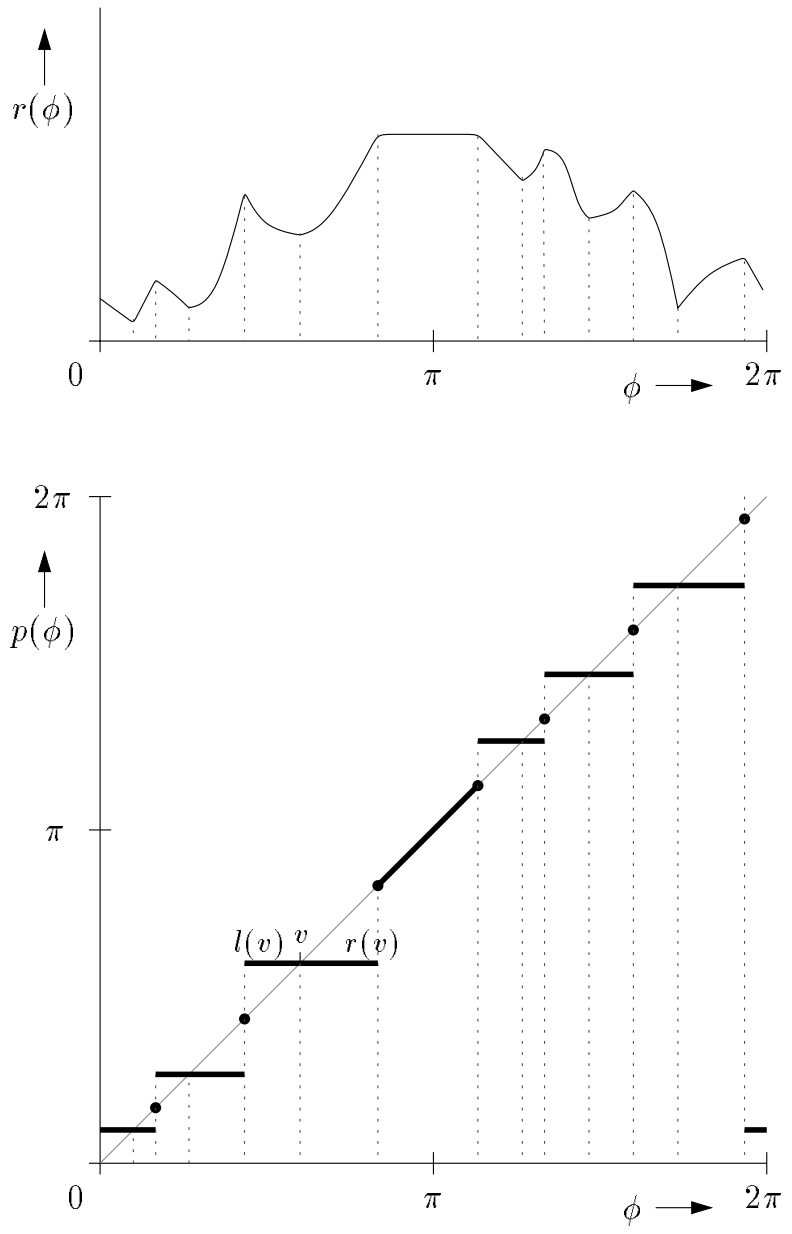

Figure 3: A radius function and the steps and ramps of the corresponding push function.

from the diameter function is accomplished in a similar manner (see [11, 17] for details).

Equilibrium orientations play a crucial role in this paper. More specifically, we use a property of the point of contact of the part $P$ with a jaw pushing $P$ in an equilibrium push direction. Let us consider a jaw pushing $P$ in a certain direction $\phi$. The jaw touches the convex part $P$ at one or infinitely many points of its boundary. Let $p$ be the rightmost of these points. We observe that, if the center-of-mass $c$ of $P$ lies to the right of the line through $p$ perpendicular to the jaw, then $c$ will rotate in clockwise direction about $p$ and towards the (supporting line of the) jaw (see Figure 4(a)). Similarly, if $c$ lies strictly to the left of the line through the leftmost contact point and perpendicular to the jaw, then $c$ will rotate in counterclockwise direction about this contact point towards the jaw. In both cases, the direction $\phi$ can impossibly be an equilibrium push direction. The part will not rotate if the 


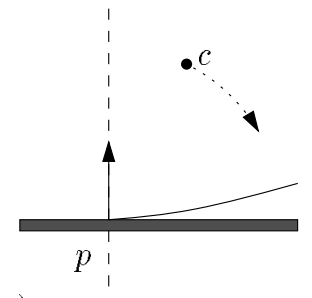

(a)

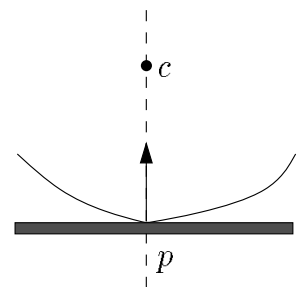

(b)

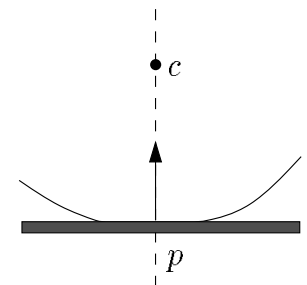

(c)

Figure 4: The center-of-mass $c$ in (a) lies to the right of the line perpendicular to the jaw and through the rightmost point of contact $p$ of the part $P$ and the jaw: the point $c$ will rotate in clockwise direction about $p$ towards the jaw. If the supporting line of the contact normal at the (in (b)) or some (in (c)) point of contact $p$ intersects $c$, then the part will not rotate when pushed in the direction of the normal; the normal direction is an equilibrium push direction.

contact normal at one of its points of contact with the jaw passes through the center-of-mass $c$ [13]. As a result, the corresponding contact or normal direction $\phi$ is an equilibrium push direction (see Figure 4(b,c)).

We use the aspect ratio of the minimum width bounding box as a measure for the eccentricity of the part. This bounding box is obtained by taking the two closest parallel supporting lines of the part and the two parallel supporting lines perpendicular to those. (The distance between its two closest parallel supporting lines is also referred to as the width of the part.) By definition, there exists no narrower bounding box of the part. The length-to-width ratio, or aspect ratio, of the minimum width bounding box can be expressed by a scalar of the form $1+\epsilon$, with $\epsilon \geq 0$. Note that the minimum bounding box is a square if $\epsilon=0$. We choose $\epsilon$ as a measure for the geometric eccentricity of a part.

Definition 1 Let $P \subset \mathbb{R}^{2}$ be a part. Let $a \geq 1$ be the aspect ratio (length-to-width ratio) of the minimum width bounding box of $P$, which is defined by the two closest parallel supporting lines and the two perpendicular supporting lines of $P$. The eccentricity $\in$ of $P$ is defined by $\epsilon=a-1$.

Figure 5 shows a part $P$. The lines $l$ and $l^{\prime}$ are the closest parallel supporting lines of $P$. Together with the two perpendicular supporting lines $m$ and $m^{\prime}$, they define the minimum width bounding box of $P$. The eccentricity $\epsilon$ of the part $P$ equals $(10.6 / 3.7)-1 \approx 1.9$.

The notion of eccentricity is commonly used to denote the ratio of the lengths of the major and minor axes of an ellipse. Observe that our notion of geometric eccentricity for parts is quite similar to the notion of eccentricity for ellipses.

\section{Grasp actions and equilibrium grasps}

Chen and Ierardi [9] present simple (push and squeeze) plans for orienting polygonal parts. Although their plans are generally not the shortest possible plans, an upper bound on the length of their plans clearly bounds the (optimum) length of plans found by Goldberg's algorithm. Chen and Ierardi's plans are based on the largest angular interval without equilibrium orientations. We use their strategy to find an upper bound on the number of pushes required 


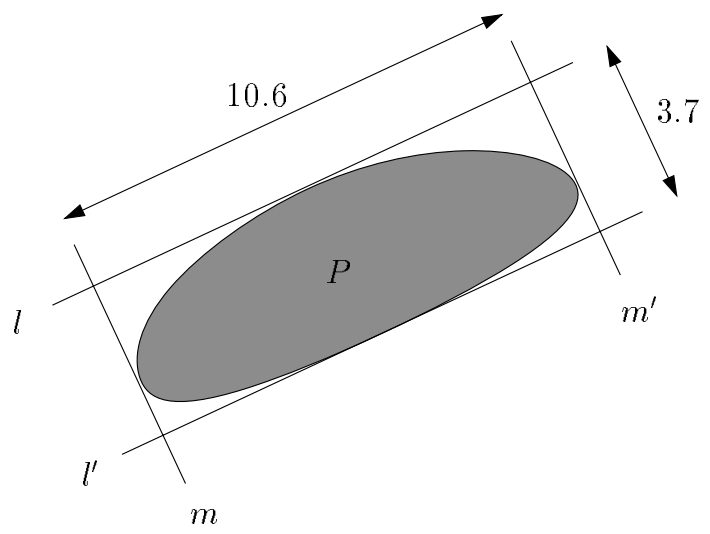

Figure 5: The closest parallel supporting lines $l$ and $l^{\prime}$ and the orthogonal supporting lines $m$ and $m^{\prime}$ define $P$ 's minimum width bounding box.

to orient an eccentric part. In a later section, we generalize the result to push-squeeze grasps and discuss some implications of part eccentricity for fence design.

Let $f$ be a transfer function of the convex part $P$. Chen and Terardi define two open intervals $l(v)=\{\phi<v \mid f(\phi)=v\}$ and $r(v)=\{\phi>v \mid f(\phi)=v\}$ for each fixed point - or equilibrium orientation - $v$ of the transfer function. Notice that an interval $l(v)$ or $r(v)$ can only be non-empty if the equilibrium orientation $v$ lies on a step of the transfer function. In that case, the interval $l(v)$ corresponds to the half-step left of $v=f(v)$ and $r(v)$ corresponds to the half-step right of $v=f(v)$ (see Figure 3). The union of all intervals $l(v)$ and $r(v)$ equals the set of all non-equilibrium orientations. Moreover, the set of all (maximal) intervals without equilibrium orientation equals the set of all intervals $l(v)$ and $r(v)$. Let $\alpha$ be the length of the longest interval in the set. We assume that $P$ has an equilibrium orientation $a$ such that $|r(a)|=\alpha$. Like Chen and Ierardi in their paper, we first focus on the case in which $P$ has no second equilibrium orientation $a^{\prime}$ satisfying $\left|r\left(a^{\prime}\right)\right|=\alpha$.

Chen and Ierardi use a sequence of equivalent basic actions to orient a polygonal part $P$ with a unique longest angular interval $r(a)$ of length $\alpha$. Each basic action consists of a reorientation of the gripper by an angle of $\alpha-\mu$, with $\mu>0$ such that $\alpha-\mu>\left|r\left(a^{\prime}\right)\right|$ for any equilibrium orientation $a^{\prime} \neq a$, and a subsequent application of the gripper. (If the longest angular interval is some $l(a)$, the same arguments will apply with reorientation by an angle of $-(\alpha-\mu)$ instead of $\alpha-\mu$.) Note that a reorientation of the gripper by $\alpha-\mu$ corresponds to a change of the orientation of the part by $\alpha-\mu$. Every basic action puts the part into an equilibrium orientation. If $P$ is a polygonal part, then the equilibrium orientations occur at isolated points in $[0,2 \pi)$. After each basic action, the part is therefore in one of a finite number of equilibrium orientations. Let us label the $m$ equilibrium orientations $a_{0}, \ldots, a_{m-1}$ in order of decreasing angle starting from $a_{0}=a$. After the first application of the gripper, the part $P$ can be in any of the equilibrium orientations $a_{0}, \ldots, a_{m-1}$. Chen and Ierardi show that every next basic action eliminates the last orientation in the sequence as possible orientation of the part. Assume that $P$ is in one of the orientations $a_{0}, \ldots, a_{k}$, for some $k \geq 1$. The key idea behind the proof is that a next basic action will cause $P$, when in $a_{0}$, to stay in $a_{0}$ because $\alpha-\mu<\left|r\left(a_{0}\right)\right|=|r(a)|$, and, when in $a_{i}$ for some $1 \leq i \leq k$, to move into some orientation $a_{j}$ with $0 \leq j \leq i-1$ because $\alpha-\mu>\left|r\left(a_{i}\right)\right|$. Upon completion of the basic 
action, the part will therefore be in one of the orientations $a_{0}, \ldots, a_{k-1}$. As a consequence, a total of $m+1$ basic actions suffices to put $P$ into orientation $a_{0}=a$. (Chen and Ierardi actually prove that $m / 2+1$ actions are sufficient by showing that every basic action reduces the number of possible stable orientations of $P$ by one.)

Our upper bound on the number of gripper applications required to orient an arbitrary convex part $P$ depends on the length $\alpha$ of the longest interval without equilibrium orientations rather than on the number of such equilibrium orientations. Another difference with the previous paragraph is that the arbitrary part $P$ can have an infinite number of equilibrium orientations as its transfer function may have ramps in addition to steps; a ramp is a continuous interval of equilibrium orientations. These modified circumstances make it impossible to discretize the problem. We modify Chen and Ierardi's proof by monitoring the length of the interval containing the possible orientations of $P$ rather than the number of possible orientations while performing the basic actions described above. We will show that two subsequent basic actions shrink the interval of possible orientations from $[b, a]$ to $[\min \{b+(\alpha-\mu), a\}, a]$. Hence, the length of the interval decreases by $\alpha-\mu$ unless $b$ is close to $a$. This leads to an upper bound of $2\lceil L / \alpha\rceil+1$ on the number of applications of the gripper, where $L$ is the period of the gripper's transfer function. (Recall that $L=\pi$ if the transfer function is a squeeze function and $L=2 \pi$ if the transfer function is a push function.)

Lemma 2 For part $P$, let $\alpha$ be the length of the unique largest angular half-interval without equilibrium orientations. The part $P$ will be oriented after $N \leq 2\lceil L / \alpha\rceil+1$ applications of the gripper, where $L$ is the period of the transfer function of the gripper.

Proof: Let $a$ again be the unique equilibrium orientation satisfying $r(a)=\alpha$. Initially, the orientation of $P$ is contained in the interval $[a+\alpha-L, a+\alpha)$. A single application of the gripper reduces the interval to $[a+\alpha-L, a]$. A first observation is that a basic action performed on a part in some equilibrium orientation $v$ causes the part to end up in an equilibrium orientation $v^{\prime}$ satisfying $v^{\prime} \geq v$. In other words, a basic action cannot move the part into a smaller equilibrium orientation.

Now let us assume that, after a number of basic actions, the part $P$ is in an equilibrium orientation $v$ in the range $[b, a]$. We may assume without loss of generality that $b$ is an equilibrium orientation. We show that after two further actions $P$ will be in an equilibrium orientation in the range $[\min \{b+(\alpha-\mu), a\}, a]$.

If $v=a$ then a reorientation of the gripper by $\alpha-\mu$ puts $P$ into orientation $a+(\alpha-\mu) \epsilon$ $r(a)$. Application of the gripper puts $P$ back into $a$. The second basic action clearly yields the same result. The final orientation $a$ of $P$ is in $[\min \{b+\alpha, a\}, a]$.

If $v \in[b, a)$ then a reorientation of the gripper by $\alpha-\mu$ puts $P$ into orientation $v+(\alpha-\mu)$ which is beyond $r(v)$ because $|r(v)|<\alpha-\mu$. We distinguish three cases:

- $v+(\alpha-\mu)$ is an equilibrium orientation.

Because $v+(\alpha-\mu)$ is an equilibrium orientation, application of the gripper will keep $P$ in $v^{\prime}=v+(\alpha-\mu)$. The second basic action puts $P$ into an orientation $v^{\prime \prime} \geq v^{\prime}=v+(\alpha-\mu)$; hence $v^{\prime \prime}$ is in $[\min \{b+(\alpha-\mu), a\}, a]$.

- $v+(\alpha-\mu) \in l\left(v^{\prime}\right)$ for some equilibrium orientation $v^{\prime}>v$.

Because $v+(\alpha-\mu) \in l\left(v^{\prime}\right)$, application of the gripper will put $P$ into $v^{\prime}>v+(\alpha-\mu)$. The second basic action puts $P$ into an orientation $v^{\prime \prime} \geq v^{\prime}>v+(\alpha-\mu)$; hence $v^{\prime \prime}$ is in $[\min \{b+(\alpha-\mu), a\}, a]$. 
- $v+(\alpha-\mu) \in r\left(v^{\prime}\right)$ for some equilibrium orientation $v^{\prime}>v$.

Because $v+(\alpha-\mu) \in r\left(v^{\prime}\right)$, application of the gripper will put $P$ into $v^{\prime}>v$. If $v^{\prime}=a$ then the second basic action keeps $P$ in $a$, which is clearly in $[\min \{b+(\alpha-\mu), a\}, a]$. If $v^{\prime}<a$, then the reorientation in the second basic action puts $P$ into orientation $v^{\prime}+(\alpha-\mu)$ which is beyond $r\left(v^{\prime}\right)$ because $\left|r\left(v^{\prime}\right)\right|<\alpha-\mu$. The orientation $v^{\prime}+(\alpha-\mu)$ is again either an equilibrium orientation itself, or in $l\left(v^{\prime \prime}\right)$ or $r\left(v^{\prime \prime}\right)$ for some equilibrium orientation $v^{\prime \prime}$, which must clearly lie beyond $r\left(v^{\prime}\right)$. Since $v+(\alpha-\mu) \in r\left(v^{\prime}\right)$, the final orientation $v^{\prime \prime}$ also lies beyond $v+(\alpha-\mu)$, so $v^{\prime \prime} \geq v+(\alpha-\mu)$; hence $v^{\prime \prime}$ is in $[\min \{b+(\alpha-\mu), a\}, a]$.

The total number of applications required to shrink the interval of possible orientations from $[a+\alpha-L, a]$ to $[a, a]$ is $2\lceil(L-\alpha) /(\alpha-\mu)\rceil \leq 2\lceil L / \alpha\rceil$. Taking into account the initial application of the gripper, we find $N \leq 2\lceil L / \alpha\rceil+1$.

Let us now drop the uniqueness assumption for the longest angular interval without equilibrium orientations and assume that there exist several equilibrium orientations $a$ with $|r(a)|=\alpha$. Assume that, after a number of basic actions, the interval of possible part orientations is reduced to $[b, a]$ where both $|r(a)|=\alpha$ and $|r(b)|=\alpha$. Chen and Ierardi prove a so-called 'stretching lemma' that deals with this problem. It reduces the interval $[b, a]$ to $[b+\delta, a](\delta>\mu)$ by means of a single application of the gripper: find an angle $\gamma$ such that $b+\gamma \in l(v)$ and $a+\gamma \in r\left(v^{\prime}\right)$ for some equilibrium orientations $v$ and $v^{\prime}$, then reorient the gripper by $\gamma$ and apply it, moving the former orientation to $v$ and the latter to $v^{\prime}$; since $v$ and $v^{\prime}$ are less far apart than $b$ and $a$, we can reorient the gripper by some appropriate angle to put $v^{\prime}$ back to $a$ and $v$ to $b+\delta$. A subsequent basic action then reduces the interval further to $[\min \{b+(\alpha-\mu), a\}, a]$. Thus we can shrink the interval of possible orientations by an application of the 'stretching lemma' followed by a basic action. The alternative strategy does not change the overall number of applications of the gripper required to orient the part.

Theorem 3 For part $P$, let $\alpha$ be the length of largest angular half-interval without equilibrium orientations. The part $P$ will be oriented after $N=2\lceil L / \alpha\rceil+1$ applications of the gripper, where $L$ is the period of the transfer function of the gripper.

Proof: By the proof of Lemma 2, we only have to consider how to shrink an interval $[b, a]$ with $|r(a)|=\alpha$ and $|r(b)|=\alpha$. Chen and Ierardi show (Lemma 2.5 [9]) that a single application of the gripper suffices to reduce the interval $[b+\delta, a](\delta>\mu)$. If the part is in orientation $v=b+\delta$, then a reorientation of the gripper will put $P$ in orientation $b+\delta+\alpha-\mu$, which is beyond $r(b)$ because $\delta>\mu$. The orientation $b+\alpha+\delta-\mu$ is either an equilibrium orientation itself, or in $l\left(v^{\prime}\right)$ or $r\left(v^{\prime}\right)$ for some equilibrium orientation $v^{\prime}$, which must clearly lie beyond the $r(b)$.

Hence, if the left endpoint $b$ of the interval of possible orientations satisfies $|r(b)|=\alpha$, then we simply replace two basic actions by one special application of the gripper plus one basic action. As a result, the total number of applications remains $2\lceil L / \alpha\rceil+1$.

Chen and Ierardi's linear bound for polygonal parts follows from Theorem 3 if one realizes that the radius function of a polygon has at most one local maximum for every vertex and at most one local minimum for every edge and that the diameter function has at most one local maximum per antipodal vertex pair and one local minimum per antipodal edge-vertex pair. In both cases we obtain a linear number of isolated equilibrium orientations, which partition 
the angular interval $[0,2 \pi)$ into a linear number of intervals without equilibrium orientations. Clearly, the length $\alpha$ of the longest of these intervals is at least a linear fraction of the full angular interval $[0,2 \pi)$. Application of Theorem 3 to this $\alpha$ yields a linear bound on $N$.

The arguments in the previous paragraph indicate that the number of equilibrium orientations directly affects the number of steps in a push or squeeze plan. A constant upper bound on the number of equilibrium orientations immediately implies a constant upper bound on the number of steps in a plan. Unfortunately, the number of equilibrium (push and squeeze) orientations can be arbitrarily large even for parts with high eccentricity $\epsilon$, even for polygonal parts. Consider the $n$-gon with high $\epsilon$ shown in Figure 6 . The vertices $v_{1}, \ldots, v_{n-1}$ lie on an

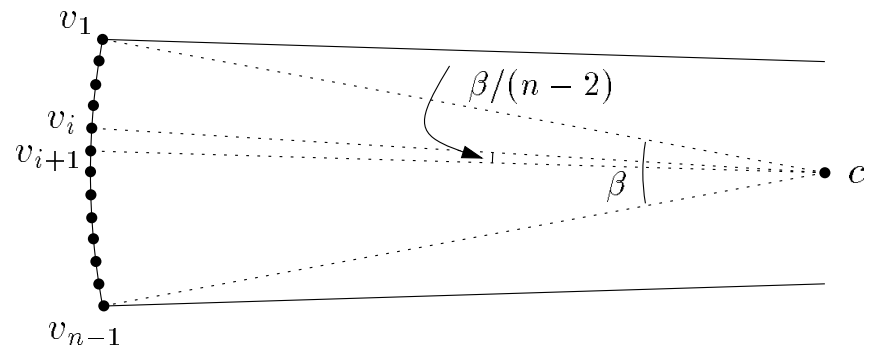

Figure 6: The extremely long and thin part can have arbitrary many equilibrium push directions.

imaginary circular arc about the center-of-mass $c$, and are assumed to be uniformly spaced. The almost parallel edges extending from $v_{1}$ and $v_{n-1}$ to the right meet at the vertex $v_{0}$. The distance from $c$ to each of the vertices $v_{i}(1 \leq i \leq n-1)$ is $r$; the angle between $c v_{1}$ and $c v_{n-1}$ equals $\beta$. The radius function of the $n$-gon has $n-1$ local maxima of $r$, which is the distance from $c$ to the tangent at $v_{i}$ perpendicular to $c v_{i}$, and $n-2$ local minima of $r \cos (\beta / 2(n-2))$, which is the distance from $c$ to the supporting line of the edge $v_{i} v_{i+1}$. The construction works for arbitrary eccentricity $\epsilon$ and for arbitrary $n$, and can easily be modified to show that the diameter function can have arbitrarily many maxima and minima. The example shows that there is no relation between eccentricity and the number of equilibrium orientations in its transfer functions. In the next section, however, we establish a relation between the eccentricity $\epsilon$ of a part and the length of the longest angular interval without equilibrium push directions, which, by applying Theorem 3 leads to a relation between the eccentricity and the required number of actions.

\section{Equilibrium push directions and part eccentricity}

As $N$ is inversely proportional to the length $\alpha$ of the longest interval without equilibrium orientation, we would like to have a lower bound on $\alpha$ given the eccentricity $\epsilon$ of the part. In this section, we derive the equivalent for push directions: an upper bound on $\epsilon$ given the length $\alpha$ of the longest interval without equilibrium push directions. In Section 5, we extend the results to push-squeeze grasps.

Consider a part $P$ with a largest angular interval without equilibrium push direction of length at most some $\alpha<\pi / 4$. There must exist an equilibrium push direction of $P$ in every interval of length $\alpha$ (otherwise, $\alpha$ would not be the upper bound on the length of the largest 
interval). We show that achieving an equilibrium push direction for $P$ in every such interval requires an upper bound on its eccentricity $\epsilon$ : the part cannot be arbitrarily thin.

Let us consider the part $P$ and its two closest parallel supporting lines. Recall that these lines are the supporting lines of the long edges of $P$ 's minimum width bounding box. Without loss of generality we assume that the distance between the closest parallel supporting lines equals 1 . It is clear that the distance from the center-of-mass $c$ to one of the two supporting lines is at most $1 / 2$. Let $l$ be this closest supporting line, and let $l^{\prime}$ be the other line. We find it convenient to express vector and push directions relative to the contact direction of $l$. The direction of any other vector $v$ equals the clockwise angle between $v$ and the positive $y$-axis (or the vertical vector pointing in upward direction). (This is basically an assumption on the orientation of the reference frame attached to $P$.) Our objective is to show that there is a bound on the extension of the part in the direction parallel to $l$ and $l^{\prime}$. (This bound almost immediately gives us a bound on the eccentricity of the part.) We achieve this objective in two steps. In a first step we consider the point of contact of the part $P$ with a line - representing a pushing jaw - touching $P$ in an equilibrium push direction from a range $[i \alpha,(i+1) \alpha]$, and show that this point must lie in a sector of the plane bounded by two half-lines (or rays) emanating from $c$. In the next step we restrict the location of this point further by constraining its position inside the sector.

We extend a collection of rays (half-lines) from the center-of-mass $c$ of $P$. The first ray $\rho_{0}$ emanates from $c$ towards and perpendicular to $l$. Additional rays $\rho_{i}$ and $\rho_{-i}(0<i \leq$ $\lfloor\pi / \alpha\rfloor$ ) are extended from $c$ in such a way that the clockwise angle from $\rho_{i}$ to $\rho_{0}$ and the counterclockwise angle from $\rho_{-i}$ to $\rho_{0}$ both equal $i \alpha$. A last ray referred to as both $\rho_{\infty}$ and $\rho_{-\infty}$ extends from $c$ towards and perpendicular to $l^{\prime}$. Figure 7 shows the resulting collection of $2\lfloor\pi / \alpha\rfloor+2$ rays. Each pair of consecutive rays $\rho_{i}$ and $\rho_{i+1}$ (for $-\lfloor\pi / \alpha\rfloor \leq i<\lfloor\pi / \alpha\rfloor$ ) bounds a sector of angle $\alpha$ from $P$ 's center-of-mass $c$. The boundary of the convex part $P$ intersects each of the sectors and each of the rays $\rho_{i}$.

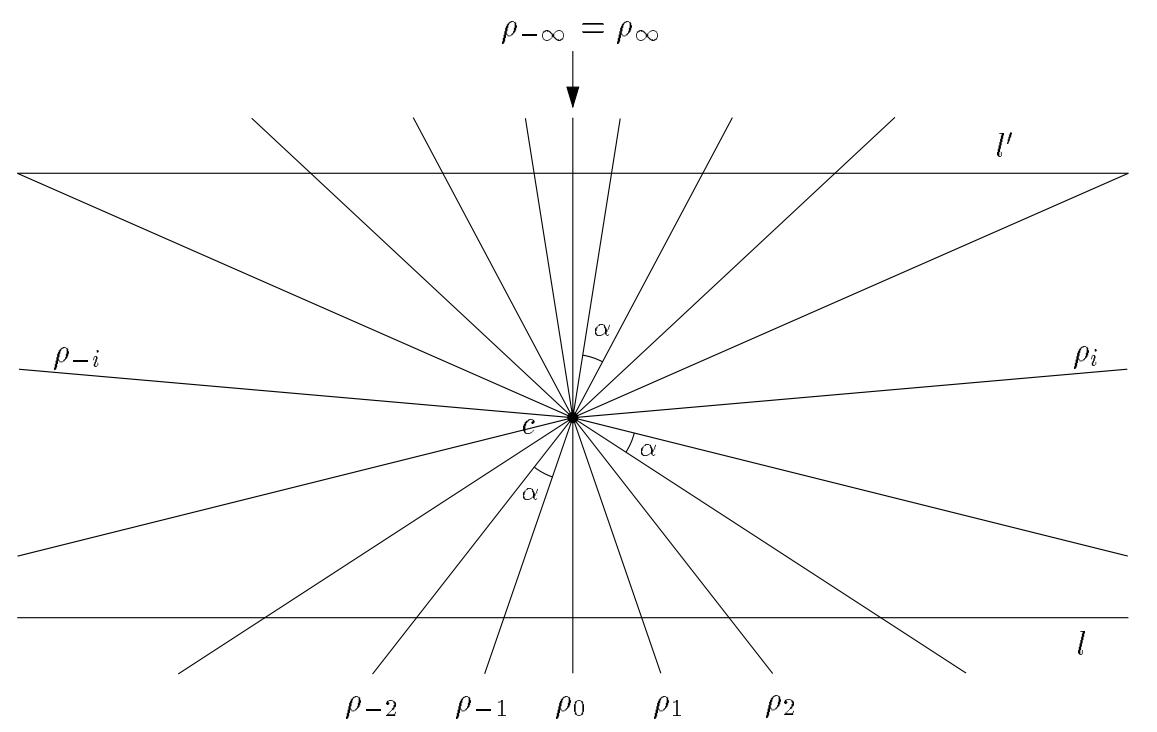

Figure 7: The $2\lfloor\pi / \alpha\rfloor+2$ rays $\rho_{i}$ emanating from $c$.

The assumption that the length of the longest interval without equilibrium orientation 
equals $\alpha$ implies that there must be an equilibrium push direction in every angular interval $[i \alpha,(i+1) \alpha]$. This in turn means that there must be a line with contact direction in $[i \alpha,(i+1) \alpha]$ such that the normal at its point of contact with the part $P$ passes through the center-ofmass $c \in P$. A simple observation is that in order for a point to have (the supporting line

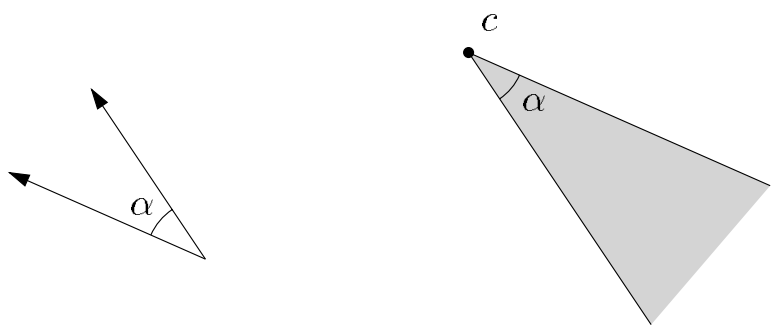

Figure 8: The point of contact of a line with contact direction in the range $[i \alpha,(i+1) \alpha]$ with the part must lie in the sector bounded by the two rays emanating from $c$ in the directions $i \alpha+\pi$ and $(i+1) \alpha+\pi$.

of ) a vector with a direction in $[i \alpha,(i+1) \alpha]$ originating from it pass through $c$ that point must lie in the wedge bounded by the supporting lines of the vectors with directions $i \alpha$ and $(i+1) \alpha$ emanating from $c$. Since the vector is not just a vector in our case but a contact normal, the point from which it originates must be a point on the boundary of $P$. Taking into account the convexity of $P$ and the fact that the center-of-mass $c$ lies inside $P$, we can constrain the position of this point to lie in the half-wedge or sector bounded by the rays emanating from $c$ in the directions $i \alpha+\pi$ and $(i+1) \alpha+\pi$ (see the shaded region in Figure 8). For any $i \in \mathrm{Z}$ satisfying $-\lfloor\pi / \alpha\rfloor \leq i<\lfloor\pi / \alpha\rfloor$ this sector is exactly the region bounded by the rays $\rho_{i}$ and $\rho_{i+1}$. Our assumption on the size of the longest interval without equilibrium orientation implies that every sector bounded by two consecutive rays $\rho_{i}$ and $\rho_{i+1}$ with $-\lfloor\pi / \alpha\rfloor \leq i<\lfloor\pi / \alpha\rfloor$ contains a point (of the boundary of $P$ ) in which a supporting line of $P$ through that point has a contact direction in the range $[i \alpha,(i+1) \alpha]$. This implied fact is powerful enough to bound the extension of the part in each of the sectors by two polygonal chains. The bounded extension of the chains in the direction parallel to the supporting lines $l$ and $l^{\prime}$ provides a bound on the extension of the part itself in that same direction, which in turn gives a bound on the eccentricity.

We define a point $p_{i}$ on every ray $\rho_{i}$ and a point $p_{-i}$ on every ray $\rho_{-i}$ for $i \geq 1$. The construction of the points is incremental in the sense that the position of $p_{i+1}$ is determined from the position of $p_{i}$, and similarly for $p_{-(i+1)}$ from $p_{-i}$. Let $p_{-1}, p_{0}$, and $p_{1}$ be the points of intersection of $l$ and $\rho_{-1}, \rho_{0}$, and $\rho_{1}$ respectively. To find $p_{i+1}$ for all $1<i<\lfloor\pi / \alpha\rfloor$, we take the line through $p_{i}$ at a counterclockwise angle of $(i-1) \alpha$ from $l$. The intersection of this line with $\rho_{i+1}$ defines $p_{i+1}$ (see Figure 9 ). The last point $p_{\infty}$ on $\rho_{\infty}$ lies at the intersection of $\rho_{\infty}$ and the line through $p_{\lfloor\pi / \alpha\rfloor}$ at a counterclockwise angle of $(\lfloor\pi / \alpha\rfloor-1) \alpha$ from $l$. The resulting chain $p_{0} p_{1} \ldots p_{\infty}$ takes a left or counterclockwise turn of $\alpha$ at every intersection with a ray $\rho_{i}$, for $i>1$. A second sequence of points $p_{-(i+1)}$ is constructed by a similar incremental scheme, using lines at a clockwise angle of $(\lfloor\pi / \alpha\rfloor-1) \alpha$ from $l$. The last point $p_{-\infty}$ on $\rho_{-\infty}=\rho_{\infty}$ coincides with $p_{\infty}$. The resulting chain $p_{0} p_{-1} \ldots p_{-\infty}$ takes a right or clockwise turn of $\alpha$ at every intersection with a ray $\rho_{-i}$. Figure 10 shows parts of the chains $p_{0} p_{1} \ldots p_{\infty}$ and $p_{0} p_{-1} \ldots p_{-\infty}$. We will show that the part $P$ lies entirely inside the region bounded by the 


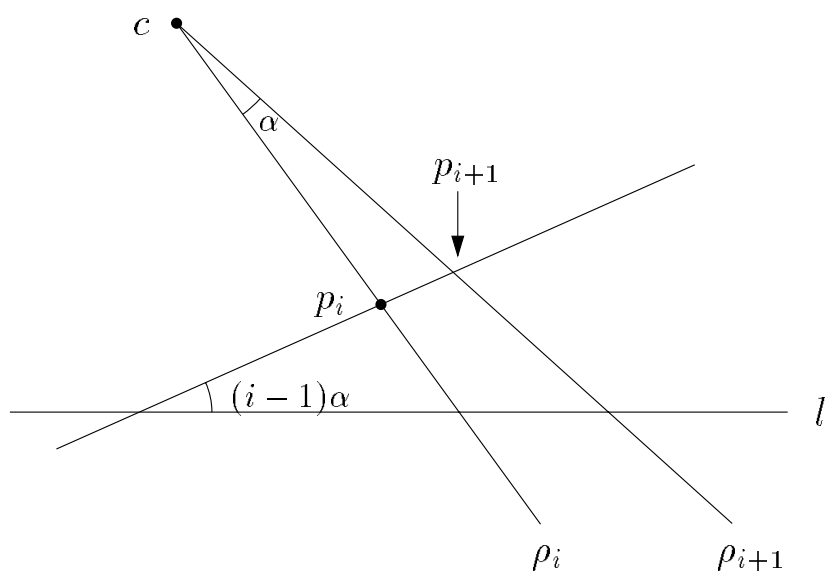

Figure 9: The point $p_{i+1}$ is the intersection of the ray $\rho_{i+1}$ and the line through $p_{i}$ at a counterclockwise angle of $(i-1) \alpha$ from $l$.

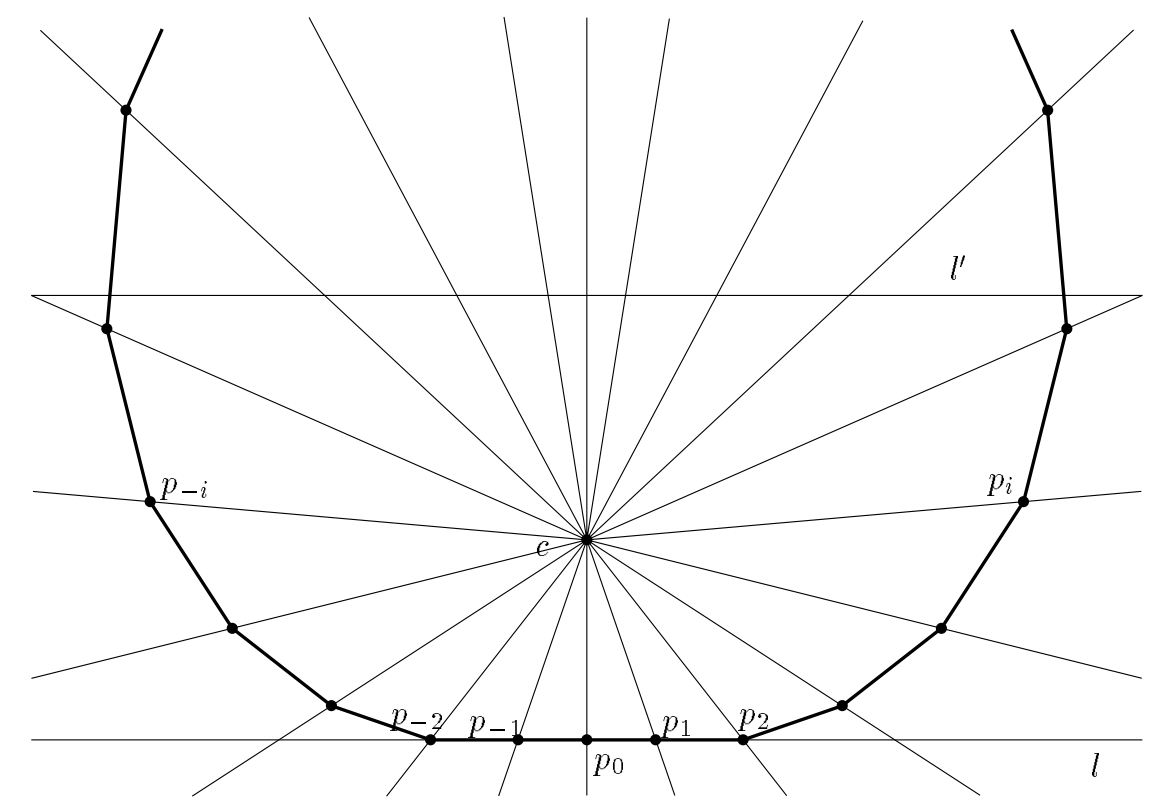

Figure 10: The chains $p_{0} p_{1} \ldots p_{\infty}$ and $p_{0} p_{-1} \ldots p_{-\infty}$ bounding the extension of the part $P$. 
chains $p_{0} p_{1} \ldots p_{\infty}$ and $p_{0} p_{-1} \ldots p_{-\infty}$.

We show by induction that the intersection of $P$ with a sector bounded by two consecutive rays $\rho_{i}$ and $\rho_{i+1}$ lies inside the triangle $c p_{i} p_{i+1}$. The intersection of $P$ with the sector between $\rho_{0}$ and $\rho_{1}$ lies inside the triangle $c p_{0} p_{1}$ simply because the intersection of that sector and the strip bounded by $l$ and $l^{\prime}$ is exactly the triangle $c p_{0} p_{1}$. Now consider a sector bounded by the rays $\rho_{i-1}$ and $\rho_{i}$ for some $1 \leq i \leq\lfloor\pi / \alpha\rfloor$ and assume that its intersection with $P$ is completely contained in the triangle $c p_{i-1} p_{i}$ (the shaded triangle in Figure 11). The triangle contains a

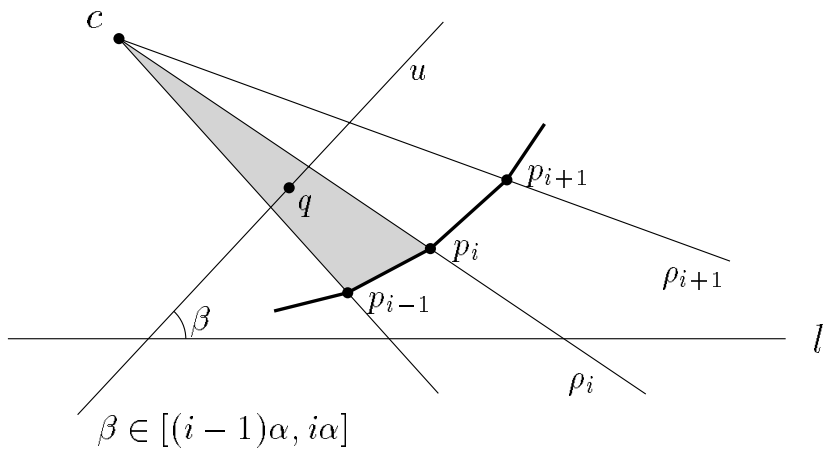

Figure 11: The tangent $u$ - which has an equilibrium contact direction in the range $[(i-1) \alpha, i \alpha]$ - intersects $\rho_{i}$ between $c$ and $p_{i}$ and $\rho_{i+1}$ between $c$ and $p_{i+1}$.

point $q$ of the boundary of $P$ in which the supporting line $u$ (of $P$ ) has a contact direction in the range $[(i-1) \alpha, i \alpha]$. As a consequence, the angle from the line $l$ to the supporting line $u$ is a counterclockwise angle in the range $[(i-1) \alpha, i \alpha]$. The location of its point of contact $q$ with $P$ and the bounds on its direction - $u$ is slightly steeper than both segments $p_{i-1} p_{i}$ and $p_{i} p_{i+1}$ - jointly imply that $u$ must intersect $\rho_{i}$ between $c$ and $p_{i}$ and must intersect $\rho_{i+1}$ between $c$ and $p_{i+1}$ (or $\rho_{\infty}$ between $c$ and $p_{\infty}$ if $i=\lfloor\pi / \alpha\rfloor$ ). As $u$ is a supporting line of the part $P, P^{\prime}$ s intersection with the sector bounded by $\rho_{i}$ and $\rho_{i+1}$ lies completely in the triangular region bounded by $\rho_{i}, \rho_{i+1}$, and $u$, which, in turn is entirely contained in the triangle $c p_{i} p_{i+1}$. As a result, the part $P$ cannot extend beyond the polygonal chain $p_{0} p_{1} \ldots p_{\infty}$. Similar arguments apply to the sectors bounded by rays $\rho_{-i}$ and $\rho_{-(i+1)}$ with $i \leq 0$, leading to the conclusion that $P$ cannot extend beyond the chain $p_{0} p_{-1} \ldots p_{-\infty}$.

The polygonal chains $p_{0} p_{1} \ldots p_{\infty}$ and $p_{0} p_{-1} \ldots p_{-\infty}$ bound the extension of the part $P$ in the direction parallel to its two closest parallel supporting lines $l$ and $l^{\prime}$. The chains also give us an upper bound on the distance $1+\epsilon$ between the two parallel supporting lines perpendicular to $l$ and $l^{\prime}$; this distance cannot exceed the distance between supporting line of $p_{0} p_{1} \ldots p_{\infty}$ perpendicular to $l$ and $l^{\prime}$ and the supporting line of $p_{0} p_{-1} \ldots p_{-\infty}$ perpendicular to $l$ and $l^{\prime}$. It is easy to determine the points of tangency of these perpendicular supporting lines. Let $k=\lceil\pi /(2 \alpha)\rceil$ and remember that the chain $p_{0} p_{1} \ldots p_{\infty}$ turns by an angle of $\alpha$ at every point $p_{i}$ with $i>1$. After $k$ turns - at $p_{k+1}$ - the chain bends back towards the common supporting line of $\rho_{0}$ and $\rho_{\infty}$ (or becomes parallel to the supporting line if $(\pi / 2) \bmod \alpha=0$ ). As a result, the point $p_{k+1}$ is the point of contact of $p_{0} p_{1} \ldots p_{\infty}$ and its supporting line perpendicular to $l$. Due to symmetry, the point of contact of the other chain and a perpendicular supporting line is $p_{-(k+1)}$. The distance between the parallel supporting lines of $p_{0} p_{1} \ldots p_{\infty}$ and $p_{0} p_{-1} \ldots p_{-\infty}$ 
perpendicular to $l$ and $l^{\prime}$ is $2\left|p_{-(k+1)} p_{k+1}\right|$ so we get

$$
1+\epsilon \leq 2\left|p_{-(k+1)} p_{k+1}\right| \text {. }
$$

Using elementary trigonometry we find

$$
\left|p_{-(k+1)} p_{k+1}\right|=\left|c p_{-(k+1)}\right| \cdot \sin (k+1) \alpha=\left|c p_{k+1}\right| \cdot \sin (k+1) \alpha .
$$

Our aim is to find the distance from $c$ to $p_{k+1}$.

We notice that all triangles $c p_{i} p_{i+1}$ with $i \geq 1$ are congruent. Each such triangle has angles $\alpha, \pi / 2+\alpha$, and $\pi / 2-2 \alpha$ at its vertices $c, p_{i}$, and $p_{i+1}$ respectively. Using the sine law we get

$$
\frac{\sin \left(\frac{\pi}{2}-2 \alpha\right)}{\left|c p_{i}\right|}=\frac{\sin \left(\frac{\pi}{2}+\alpha\right)}{\left|c p_{i+1}\right|}
$$

for all $i \geq 1$, which is equivalent to

$$
\left|c p_{i+1}\right|=\frac{\cos \alpha}{\cos 2 \alpha} \cdot\left|c p_{i}\right| .
$$

for all $i \geq 1$. Along with the obvious

$$
\left|c p_{1}\right|=\frac{\left|c p_{0}\right|}{\cos \alpha}
$$

we obtain

$$
\left|c p_{i}\right|=\frac{\cos ^{i-2} \alpha}{\cos ^{i-1} 2 \alpha} \cdot\left|c p_{0}\right|,
$$

for all $i \geq 1$. Note that, by its construction, the point $p_{0}$ is the point on $l$ closest to $c$, so

$$
\left|c p_{0}\right| \leq 0.5
$$

We can combine Equations (1) - (4) to find an upper bound on the eccentricity $\epsilon$ in terms of the upper bound of $\alpha$ on the length $\alpha$ of the largest interval without equilibrium orientations:

$$
\begin{aligned}
\epsilon & \leq 2\left|p_{-(k+1)} p_{k+1}\right|-1 \\
& =2\left|c p_{k+1}\right| \cdot \sin (k+1) \alpha-1 \\
& =2\left|c p_{0}\right| \cdot \frac{\cos ^{k-1} \alpha \cdot \sin (k+1) \alpha}{\cos ^{k} 2 \alpha}-1 \\
& \leq \frac{\cos ^{k-1} \alpha \cdot \sin (k+1) \alpha}{\cos ^{k} 2 \alpha}-1,
\end{aligned}
$$

where $k=\lceil\pi /(2 \alpha)\rceil$. Theorem 4 summarizes the result.

Theorem 4 Let $P$ be a part and let $\alpha$ be the length of the longest angular interval without equilibrium push directions. Then $P$ 's eccentricity $\epsilon$ is bounded by

$$
\epsilon \leq \frac{\cos ^{k-1} \alpha \cdot \sin (k+1) \alpha}{\cos ^{k} 2 \alpha}-1,
$$

where $k=\lceil\pi /(2 \alpha)\rceil$. 


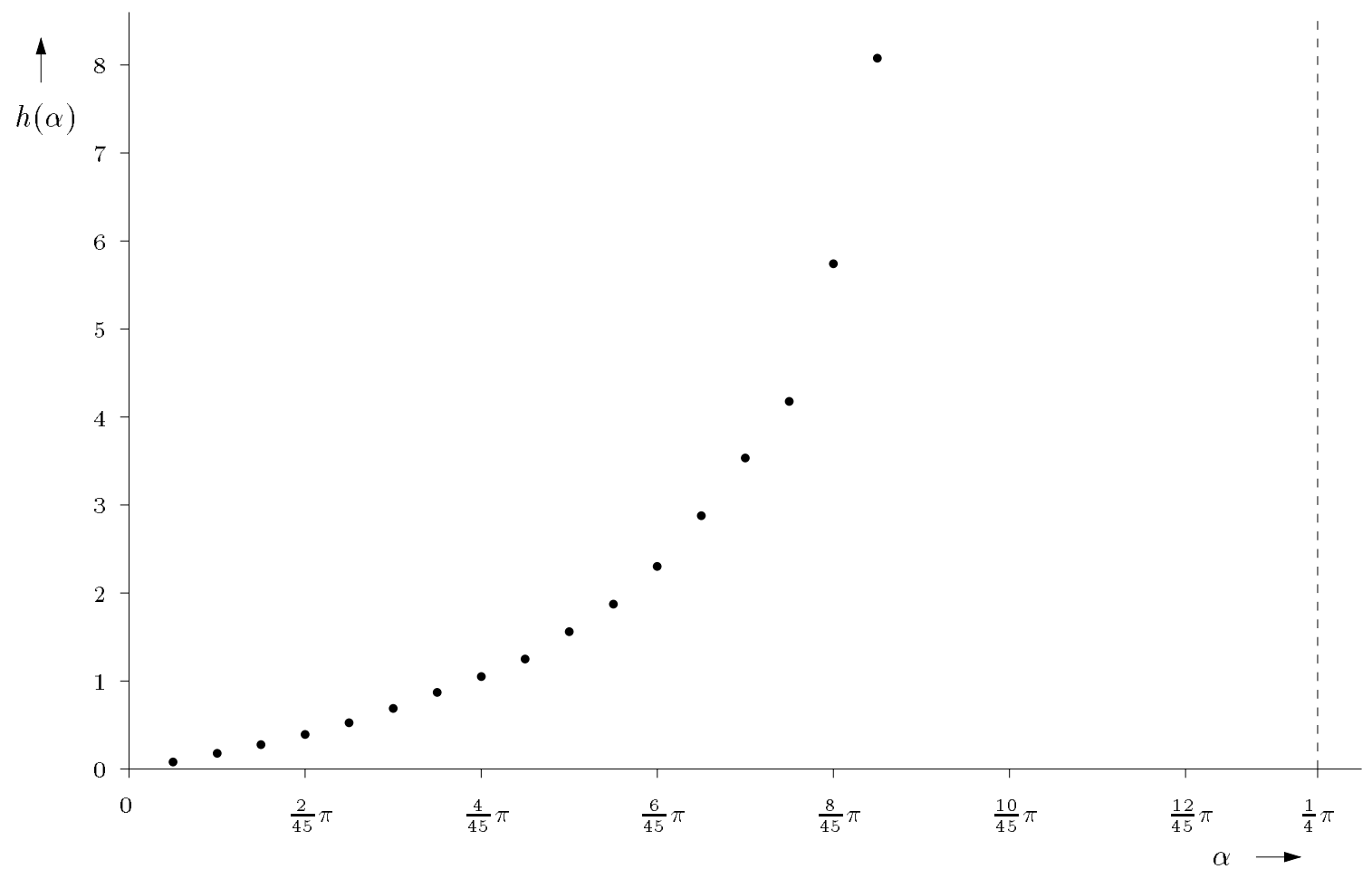

Figure 12: Upper bound $h(\alpha)$ on the eccentricity $\epsilon$ for various bounds $\alpha$ on the length of the longest interval without equilibrium push direction.

Thus we have derived an upper bound on $\epsilon$ given an upper bound on $\alpha$, which is equivalent to a lower bound on $\alpha$ given a lower bound on $\epsilon$. Figure 12 shows the values of

$$
h(\alpha)=\frac{\cos ^{k-1} \alpha \cdot \sin (k+1) \alpha}{\cos ^{k} 2 \alpha}-1,
$$

with $k=\lceil\pi /(2 \alpha)\rceil$, for the even multiples $i \in(0,45)$ of $\pi / 180$ radians.

In order to combine Theorems 3 and 4 into an upper bound on the number of pushes $N$ as a function of the eccentricity $\epsilon$, we would have to invert the function $h$ given above. Given its complexity and its discontinuity, inverting $h$ seems impossible. Therefore, we settle for the less elegant formulation of the bound on the length of the push plan as a function of the part eccentricity given by Theorem 5 .

Theorem 5 Let $P$ be a part with eccentricity

$$
\epsilon>\frac{\cos ^{k-1} \alpha \cdot \sin (k+1) \alpha}{\cos ^{k} 2 \alpha}-1
$$

$(k=\lceil\pi /(2 \alpha)\rceil)$, for some $\alpha \in(0, \pi / 4)$. Then, $P$ can be oriented by a push plan of length $N$ with

$$
N \leq 2\left\lceil\frac{2 \pi}{\alpha}\right\rceil+1
$$


Theorem 5 shows that the number of push actions needed to orient a part is a function of its eccentricity. It provides the first bound on the length of a push plan for non-polygonal parts, and improves the bound of $2 n-1$ by Chen and Ierardi for a polygonal part with $n$ vertices to the minimum of $2 n-1$ and the bound given by Theorem 5 based on the part's eccentricity. We will see below that the bound on the number of actions provided by Theorem 5 is relatively low even for smaller values of $\epsilon$.

Figure 13 shows upper bounds on the length of push plans for parts of various degrees of eccentricity. The values are compiled from the data points of Figure 12 and Theorem 3. As a result, there may exist tighter lower bounds on the eccentricity for some push plan lengths $N$. The objective of Figure 13, however, is to provide some indication of the relation between the values of $\epsilon$ and $N$. The figure shows that even for parts with low eccentricity the length

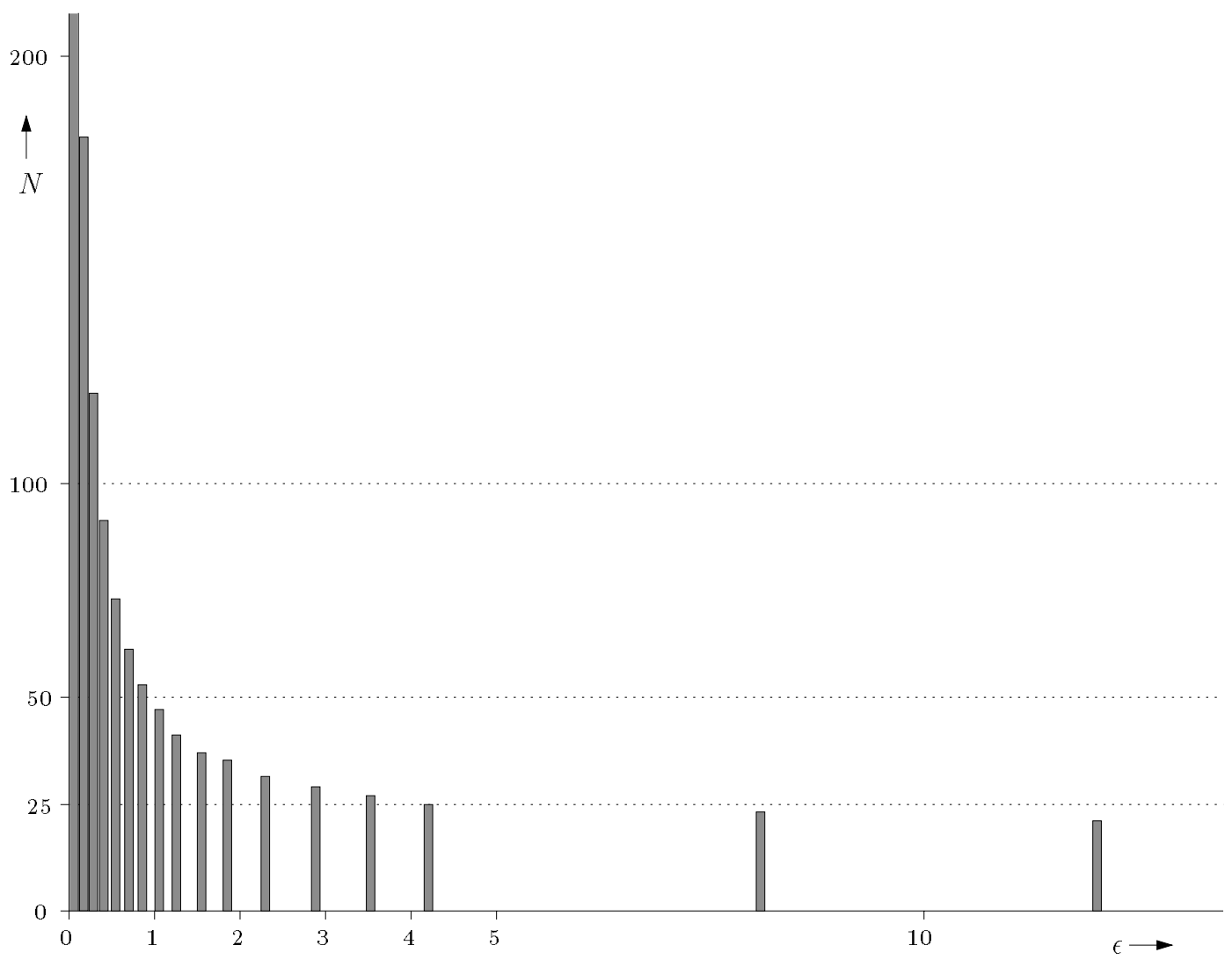

Figure 13: Upper bound on the length $N$ of an optimal push plan versus the eccentricity $\epsilon$ of the part to be oriented.

of the push plan is bounded by some relatively low constant. Moreover, we may expect the length of the shortest push plan to remain far below the upper bound provided by Theorem 5 - which actually bounds the length of some rigorous (non-optimal) push plan. (Note that the bound will never fall below 19, even though parts with very high eccentricity can easily be oriented by less than a handful of pushes.) 


\section{Extension to push-squeeze grasps and fence design}

In this section, we extend the results of the previous section to push-squeeze actions. Recall that a part is now pushed by a single jaw until it settles, and then squeezed by two jaws. As a consequence, the final orientation of a part in initial orientation $\phi$ will be $s(p(\phi))=(s \circ p)(\phi)$.

Let $f$ and $f^{\prime}$ be two generic transfer functions, and let $\alpha$ be the length of the longest angular interval without equilibrium orientation of the function $f$. Lemma 6 bounds the length of the longest interval without equilibrium orientation of the composite transfer function $f^{\prime} \circ f$.

Lemma 6 Let $f$ and $f^{\prime}$ be transfer functions and let $\alpha$ be the length of the longest angular interval without equilibrium orientation of $f$. Then, the longest interval without equilibrium orientation of $f^{\prime} \circ f$ has length at least $\alpha / 2$.

Proof: Let $I$ be an interval without equilibrium orientations of $f$ with length $\alpha$. Recall that $I$ is either of the form $l(v)=\{\phi<v \mid f(\phi)=v\}$ or of the form $r(v)=\{\phi>v \mid f(\phi)=v\}$ for some fixed point $v$. In any case, we get that $f(\phi)=v$ for all $\phi \in I$. Let $w=f^{\prime}(v)$. Clearly, we obtain that $f^{\prime}(f(\phi))=w$ for all $\phi \in I$. Hence, there exists an interval $J \supseteq I$ such that $\left(f^{\prime} \circ f\right)(\phi)=w$ for all $\phi \in J$ and some fixed point $w$ of $f^{\prime} \circ f$. In other words, $f^{\prime} \circ f$ has a step of length $|J| \geq|I| \geq \alpha$. It is clear that one of the two half-steps of this step must have length at least $|J| / 2 \geq \alpha / 2$.

Lemma 6 implies that if $\alpha$ is the length of the longest interval without equilibrium push direction of a part $P$, then the length of its longest interval without equilibrium push-squeeze orientation is at least $\alpha / 2$. Theorem 3 yields that this part $P$ can be oriented by $N \leq$ $2\lceil 4 \pi / \alpha\rceil+1$ push-squeeze actions. As a consequence, the length of the shortest push-squeeze plan for a part $P$ is at most (approximately) twice as long as the upper bound on the length of the shortest push plan for $P$. Note that this is under worse-case conditions. In practice, the composition tends to increase the lengths of the intervals without equilibrium orientations, thereby reducing $N$. Theorem 7 summarizes the result for push-squeeze plans.

Theorem 7 Let $P$ be a part with eccentricity

$$
\epsilon>\frac{\cos ^{k-1} \alpha \cdot \sin (k+1) \alpha}{\cos ^{k} 2 \alpha}-1
$$

$(k=\lceil\pi /(2 \alpha)\rceil)$, for some $\alpha \in(0, \pi / 4)$. Then, $P$ can be oriented by a push-squeeze plan of length $N$ with

$$
N \leq 2\left\lceil\frac{4 \pi}{\alpha}\right\rceil+1 .
$$

We conclude that a constant number of push-squeeze actions suffices to orient parts with non-zero eccentricity.

A different type of part feeder aligns parts as they move down a conveyor belt and slide along a sequence of fences placed along the belt. It has been noted $[5,20]$ that finding a sequence of fences is equivalent to finding a sequence of push actions under the constraint that every reorientation of the jaw imposes a restriction on the next reorientation. In other words, the previous push angle offset restricts the next offset. More specifically, if the $i$-th reorientation is in the range $(0, \pi / 2) \cup(-\pi,-\pi / 2)$ then the $i+1$-th reorientation must be in 
$(0, \pi / 2) \cup(\pi / 2, \pi)$, and if the $i$-th reorientation is in $(-\pi / 2,0) \cup(\pi / 2, \pi)$ then the $i+1$-th reorientation must be in $(-\pi / 2,0) \cup(-\pi,-\pi / 2)$. The restrictions learn that a sequence of either (exclusively) clockwise reorientations or (exclusively) counterclockwise reorientations smaller than $\pi / 2$ can be implemented by a sequence of fences. A part with a unique largest angular interval without equilibrium orientations of length $\alpha<\pi / 2$ can therefore be oriented by a sequence of fences of length at most $\lceil 4 \pi / \alpha\rceil+1$. If the part has a certain positive eccentricity, then this upper bound turns into a constant.

Akella et al. [2] explore part orienting by a sensorless part feeder (1JOC) consisting of a conveyor belt and a single rotational fence. The fence repeatedly catches the part after which it uses its rotational degree of freedom to reorient the part while transporting it back along the belt. The authors show that the effect of a single catch followed by a reorientation of the part is equivalent to a single push followed by a reorientation of the jaw. As the rotational fence can accomplish any part reorientation, any push plan can be implemented by sequence of catches and fence rotations of equal length. Theorem 4 therefore bounds the number of catches and fence rotations needed to orient a part with eccentricity $\epsilon$.

\section{Conclusion}

We have presented new bounds on the number of actions required to orient parts without sensors. We formalized the intuitive description of parts as 'fat' or 'thin' based on eccentricity and showed that only $O(1)$ actions are required for parts with non-zero eccentricity. The analysis also applies to curved parts, providing the first complexity bound for non-polygonal parts. Our results also yield constant bounds on part feeders that use fences and conveyor belts. Similar results may be possible for part orienting on a motion array $[6,7]$.

Complexity bounds for algorithms for robotic motion and manipulation can be misleading when they are constructed with pathological 'worst-case' scenarios that rarely appear in practice. Complexity can in some cases be reduced by characterizing non-pathological objects in terms of intuitive geometric properties. Examples include computational geometric results in robot motion planning, hidden surface removal, depth orders, motion planning, point location, and range searching, where 'fatness' reduces combinatorial $[3,14,18]$ and algorithmic $[1,4,12,15,19]$ complexities. In the same spirit we prove a symmetrical result: for part orienting, thinness reduces complexity.

\section{Acknowledgements}

We thank Matt Mason, Kevin Lynch, Srinivas Akella, and Robert-Paul Berretty for useful feedback. This research was supported by NATO Collaborative Research Grant CRG 951224. Van der Stappen is supported by the Netherlands Organization for Scientific Research (N.W.O.). Goldberg's research is supported in part by the National Science Foundation under IRI-9612491 and by Presidential Faculty Fellow Award IRI-9553197.

\section{References}

[1] P.K. Agarwal, M.J. Katz, and M. Sharir, Computing depth orders for fat objects and related problems, Computational Geometry: Theory and Applications 5 (1995), pp. 187-206. 
[2] S. Akella, W. Huang, K.M. Lynch, and M.T. Mason, Sensorless parts feeding with a one joint robot, in: Algorithms for Robotic Motion and Manipulation (J.-P. Laumond and M. Overmars (Eds.)), A.K. Peters (1996), pp. 229-238.

[3] H. Alt, R. Fleischer, M. Kaufmann, K. Mehlhorn, S. Näher, S. Schirra, AND C. Uhrig, Approximate motion planning and the complexity of the boundary of the union of simple geometric figures, Algorithmica 8 (1992), pp. 391-406.

[4] M. DE Berg, Linear size binary space partitions for fat objects, Proc. 3rd Annual European Symp. on Algorithms (ESA'95), Lecture Notes in Computer Science 979 (1995), pp. 252-263.

[5] R.-P. Berretty, K.Y. Goldberg, M.H. Overmars, and A.F. van der Stappen, A graph search approach to passive fence design, in preparation.

[6] K.-F. Böhringer, V. Bhatt, and K.Y. Goldberg, Sensorless manipulation using transverse vibrations of a plate, Proc. IEEE Int. Conf. on Robotics and Automation, Nagoya, Japan (1995), pp. 1989-1996.

[7] K.-F. Böhringer, B.R. Donald, and N.C. MacDonald, Upper and lower bounds for programmable vector fields with applications to MEMS and vibratory plate part feeders, in: Algorithms for Robotic Motion and Manipulation (J.-P. Laumond and M. Overmars (Eds.)), A.K. Peters (1996), pp. 255-276.

[8] M. Brokowski, M. Peshinin, and K.Y. Goldberg, Curved fences for part alignment, Proc. IEEE Int. Conf. on Robotics and Automation, Atlanta GA (1993), Vol. 3, pp. 467473.

[9] Y.-B. Chen and D.J. Ierardi, The complexity of oblivious plans for orienting and distinguishing polygonal parts, Algorithmica 14 (1995), pp. 367-397.

[10] M.A. Erdmann and M.T. Mason, An exploration of sensorless manipulation, IEEE Journal of Robotics and Automation 4 (1988), pp. 369-379.

[11] K.Y. Goldberg, Orienting polygonal parts without sensors, Algorithmica 10 (1993), pp. 201-225.

[12] M.J. Katz, M.H. Overmars, and M. Sharir, Efficient hidden surface removal for objects with small union size, Computational Geometry: Theory and Applications 2 (1992), pp. 223-234.

[13] M.T. MAson, Manipulator grasping and pushing operations, AI-TR-690, PhD Thesis, MIT (1982).

[14] J. Matoušek, J. Pach, M. Sharir, S. Sifrony, and E. Welzl, Fat triangles determine linearly many holes, SIAM Journal on Computing 23 (1994), pp. 154-169.

[15] M.H. Overmars and A.F. van der Stappen, Range searching and point location among fat objects, Journal of Algorithms 21 (1996).

[16] M.A. Peshin and A.C. Sanderson, Planning robotic manipulation strategies for workpieces that slide, IEEE Journal on Robotics and Automation 4 (1988), pp. 524-531. 
[17] A.S. RAO AND K.Y. GoldBERG, Manipulating algebraic parts in the plane, IEEE Transactions on Robotics and Automation 11 (1995), pp. 598-602.

[18] A.F. van der Stappen, D. Halperin, M.H. Overmars, The complexity of the free space for a robot moving amidst fat obstacles, Computational Geometry: Theory and Applications 3 (1993), pp. 353-373.

[19] A.F. van Der Stappen and M.H. Overmars, Motion planning amidst fat obstacles, Proc. 10th Ann. ACM Symp. on Computational Geometry (1994), pp. 31-40.

[20] J. Wiegley, K. Goldberg, M. Peshin, and M. Brokowski, A complete algorithm for designing passive fences to orient parts, Proc. IEEE International Conf. on Robotics and Automation, Minneapolis MN (1996), pp. 1133-1139. 